\title{
Cystic Fibrosis Transmembrane Conductance Regulator Mutations That Disrupt Nucleotide Binding
}

\author{
James Logan, * David Hiestand, ${ }^{*}$ Paru Daram, Zhen Huang, Donald D. Muccio, ${ }^{\mathbf{5}}$ John Hartman, \\ Boyd Haley, "William J. Cook," and Eric J. Sorscher \\ ${ }^{\ddagger}$ Department of Biochemistry University of Kentucky Lexington, Kentucky 40536; and Departments of Medicine, Physiology and \\ Biophysics, *Pediatrics, "Pathology, "Chemistry, and "Center for Macromolecular Crystallography, University of Alabama \\ at Birmingham, Birmingham, Alabama 35294
}

\begin{abstract}
Increasing evidence suggests heterogeneity in the molecular pathogenesis of cystic fibrosis (CF). Mutations such as deletion of phenylalanine at position $508(\Delta F 508)$ within the cystic fibrosis transmembrane conductance regulator (CFTR), for example, appear to cause disease by abrogating normal biosynthetic processing, a mechanism which results in retention and degradation of the mutant protein within the endoplasmic reticulum. Other mutations, such as the relatively common glycine $\rightarrow$ aspartic acid replacement at CFTR position 551 (G551D) appear to be normally processed, and therefore must cause disease through some other mechanism. Because $\Delta F 508$ and G551D both occur within a predicted nucleotide binding domain (NBD) of the CFTR, we tested the influence of these mutations on nucleotide binding by the protein. We found that G551D and the corresponding mutation in the CFTR second nucleotide binding domain, G1349D, led to decreased nucleotide binding by CFTR NBDs, while the $\Delta$ F508 mutation did not alter nucleotide binding. These results implicate defective ATP binding as contributing to the pathogenic mechanism of a relatively common mutation leading to $\mathrm{CF}$, and suggest that structural integrity of a highly conserved region present in over $\mathbf{3 0}$ prokaryotic and eukaryotic nucleotide binding domains may be critical for normal nucleotide binding. (J. Clin. Invest. 1994. 94:228-236.) Key words: cystic fibrosis • genetics - pathogenesis - ATP • structure
\end{abstract}

\section{Introduction}

Many of the mutations which cause clinical cystic fibrosis (CF ${ }^{1}$ occur within the two nucleotide binding domains of the cystic fibrosis transmembrane conductance regulator (CFTR) (1-3). While the precise role of the nucleotide binding domains (NBDs) in CFTR function is still unknown, previous studies

Address correspondence to E. J. Sorscher, Department of Physiology and Biophysics, University of Alabama at Birmingham, UAB Station, Birmingham, AL 35294-0005.

Received for publication 16 June 1993 and in revised form 21 December 1993.

1. Abbreviations used in this paper: $\mathrm{ABC}$, ATP binding cassette; $\mathrm{CF}$, cystic fibrosis; CFTR, CF transmembrane regulator; GST, glutathione$s$-transferase; NBD, nucleotide binding domain.

J. Clin. Invest.

(C) The American Society for Clinical Investigation, Inc. 0021-9738/94/07/0228/09 \$2.00

Volume 94 , July 1994, 228-236 have shown that the anion channel function of CFTR is dependent upon the presence of ATP or other nucleotides (4-8). Studies in our laboratory $(9)$ as well as by others $(10,11)$ have suggested that $\Delta F 508$, the most common mutation associated with clinical $\mathrm{CF}$, is not associated with disrupted nucleotide binding by folded CFTR polypeptides. Phenylalanine 508 is not highly conserved among the NBDs of more than 30 prokaryotic and eukaryotic members of the ATP binding cassette (ABC) gene family which includes CFTR $(1,12,13)$; this observation has suggested that the F508 residue might not directly contribute to ATP binding by CFTR. In addition, increasing evidence suggests that CFTR possessing the $\triangle F 508$ mutation is improperly processed within epithelial cells, and that disease results because the mutant protein fails to insert properly in the apical cell membrane (14-17). Such a pathogenic mechanism is not likely to be dependent upon ATP binding by the NBD.

The second most common mutation leading to clinical cystic fibrosis is a glycine to aspartic acid replacement within NBD-1 at CFTR position 551 (G551D) $(2,18,19)$. Because (a) both G551D and the corresponding mutation in NBD-2, G1349D, cause clinical CF; $(b)$ both mutations involve a glycine residue which is invariant in alignments of $>30 \mathrm{nu}-$ cleotide binding domains from the ATP binding cassette gene family $(1,12,13) ;(c)$ chloride channel studies from heterologous eukaryotic cells expressing recombinant CFTR have raised the possibility of a defective response to ATP by CFTR with these mutations (5); and (d) both G551D and G1349D appear to be normally processed to the cell membrane, $(5,14$, 15 ), we tested the influence of these mutations on nucleotide binding by CFTR NBDs. We found that G551D and G1349D, but not $\Delta F 508$, significantly decreased nucleotide binding by recombinant NBDs. These results directly demonstrate abnormal nucleotide binding as the result of CFTR mutations, and may have important implications concerning the general structure of nucleotide binding domains within the ABC gene family.

\section{Methods}

Recombinant synthesis and purification of CFTR nucleotide binding domains. A series of polymerase chain reaction (PCR) primers defining CFTR NBD-1 (AA426 to 588, 5'GCGCGAATTCATGACAGCCTCTTCTTCAG3' and 5'GCCATCAGTTTACAGACACAGAATTCAAA $3^{\prime}$ ) or NBD-2 (amino acids 1219 to $1387,5^{\prime}$ 'GCGCGAATTCAATACACAGAAGGTGGAAATGCC ${ }^{\prime}$ ' and 5'GTGCAATCAGCAAATGCTTGTTTTAGAATTCTTC $3^{\prime}$ ) were used to amplify the respective wildtype and mutant NBDs from CFTR cDNA plasmid templates obtained from the American Tissue Culture Collection (Rockville, MD) (wild type: ATCC no. T16-1 and $\triangle$ F508: ATCC no. C1-1/5) or as a generous gift from Alan Smith and Richard Gregory (Genzyme Corp., Cambridge, MA) (full length CFTR cDNAs containing G551D or G1349D) $(4,5$, 15). PCR conditions were $1 \mathrm{~min} \times 94^{\circ} \mathrm{C}$ denaturation, $2 \mathrm{~min} \times 50^{\circ} \mathrm{C}$ 

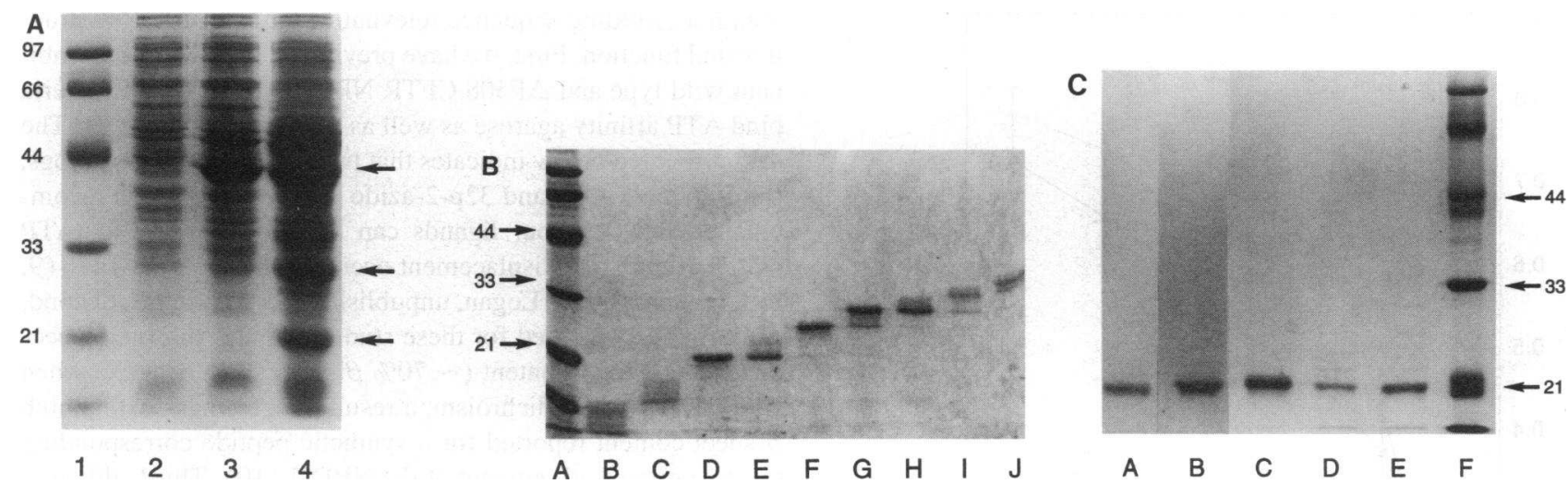

Figure 1. Synthesis and purification of recombinant CFTR NBD-1 and NBD-2. $(A)$ CFTR NBDs 1 and 2 were synthesized as fusion proteins with GST and induced using isopropyl $\beta$-D-thiogalactopyranoside (Sigma Chemical Co.) (IPTG), according to a previously described protocol (9). Lanes 2 and 3 show total $E$. coli cell lysate prior to and after IPTG induction, respectively (upper arrow, GST/wild type NBD-1 fusion protein). Lane 4, following dialysis into a protease cleavage buffer, human thrombin was used to cleave the fusion protein into its two constituents; a 28$\mathrm{kD}$ glutathione-s-transferase fragment (middle arrow) and the 21-kD wild-type NBD-1 (lowest arrow). Lane 1 contains molecular mass standards. $(B)$ Cleaved material such as that shown in panel $A$., lane 4 was loaded on a preparative electrophoresis cell (Bio Rad). Proteins were separated on the basis of molecular mass and collected in 5-ml fractions as they eluted from the bottom of the gel. Lanes $B-J$ are $20-\mu \mathrm{l}$ samples from every fifth fraction. The purified NBD-1 $(21 \mathrm{kD})$ is seen in lanes $D$ and $E$. Molecular mass standards are shown in lane $A$. ( $C$ ) Using the same approach, a series of wild-type and mutant NBDs were purified. Lane $A$, wild-type NBD-1; lane $B, \triangle$ F508 NBD-1; lane $C$, G551D NBD-1; lane $D$, wildtype NBD-2; lane $E$, G1349D NBD-2; lane $F$, molecular mass standards. Gels in $A-C$ contain $12 \%$ acrylamide. GST/NBD fusion proteins run aberrantly on $12 \%$ SDS-PAGE $(\sim 43 \mathrm{kD})$. This has been noted for all five independently cloned NBD polypeptides used in this study, as well as for four additional full length NBD mutants (not shown).

annealing, and $3 \min \times 72^{\circ} \mathrm{C}$ elongation. Primers were chosen to create EcoRI sites at the ends of PCR products to allow cloning into the EcoRI site of pGEX 2T (Pharmacia Fine Chemicals, Piscataway, NJ) in frame with glutathione-s-transferase (GST) and immediately $3^{\prime}$ to an engineered thrombin cleavage site (9). All PCR products obtained by this method were of the predicted size (524-bp NBD-1, 545-bp NBD-2). In addition, identity of correct recombinants were verified by $(a)$ restriction mapping using 10-12 digests per recombinant construct; $(b)$ re-amplification of the full length NBD insert using the recombinant plasmid as template; $(c)$ recombinant plasmid-directed synthesis of fusion protein and specific cleavage by human thrombin into the two predicted fragments; and $(d)$ amino-terminal sequencing and compositional analysis (13 amino-terminal residues of NBD-1 and 12 amino-terminal residues of NBD-2, as well as $>30$ internal residues obtained during peptide mapping matched the predicted sequence of the recombinant proteins).

Recombinant fusion protein was collected and partially purified as an inclusion body preparation as described previously (9). After thrombin treatment (in a protease cleavage buffer containing $50 \mathrm{mM}$ Tris $\mathrm{HCl}$,

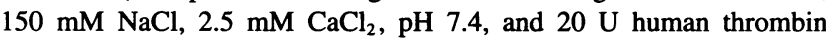
(Sigma Chemical Co., St. Louis, MO)/10 mg recombinant protein) $\sim 3$ $\mathrm{mg}$ of the partially purified, cleaved preparation (i.e., containing NBD, GST, some uncleaved fusion protein and minor bacterial contaminants) was applied to the top of a preparative acrylamide gel (Bio Rad Laboratories) and the NBD purified using electrophoresis through $12 \%$ acrylamide as in Fig. $1 \mathrm{~B}$. The observation that the polyacrylamide gel electrophoresis based protein purification step used here required solubilization in $0.8 \%$ SDS is consistent with a recent report that solubilization of recombinant full length CFTR was refractory to a large series of detergents, but ultimately could be solubilized in 2\% SDS while still permitting efficient $(20-40 \%)$ refolding of the protein into a native form capable of supporting CFTR anion channel activity in the planar lipid bilayer (6).

Trinitrophenyl ATP binding by NBDs. $250 \mu \mathrm{g}$ per ml purified NBD$1(\sim 10 \mu \mathrm{M})$ in $10 \mathrm{mM}$ Tris, $\mathrm{pH} 7.4$, was incubated at $4^{\circ} \mathrm{C}$ with increasing concentrations of trinitrophenyl ATP (TNP-ATP; Molecular Probes, Inc., Eugene, OR). TNP-ATP demonstrates fluorescence enhancement when present in a nucleotide binding pocket $(10,11)$. Adenine nucleotide fluorescence was measured at each data point for $1 \mathrm{~s}$ in a 1-ml quartz cuvette using a Perkin Elmer LS-50 spectrofluorometer (Perkin-Elmer Corp., Norwalk, CT) with excitation $408 \mathrm{nM}$ and emission $538 \mathrm{nM}$. Fluorescence enhancement was determined at each data point by measuring $(a)$ the fluorescent signal of buffer plus TNP-ATP at each concentration studied; $(b)$ the fluorescence of protein in buffer alone; and $(c)$ the fluorescent signal of protein + TNP-ATP at each concentration and using the calculation: fluorescence enhancement $=($ fluorescence signal of protein + TNP-ATP $)-($ fluorescence of TNPATP alone + fluorescence of protein alone). Because preliminary experiments indicated that binding of TNP-ATP to the NBD-1 is not dependent upon divalent cations, $\mathbf{M g}^{2+}$ was not added to the titration solutions.

$\gamma 32 p$-2-azido ATP labeling of NBDs. 2-azido ATP was synthesized from 2 azido AMP (20) by the method of Michelson as modified by Salvucci et al. (21). [ $\gamma$-32p]2-azido ATP (sp. act. 5-35 mCi/ $\mu \mathrm{mol}$ ) was then prepared and purified as described previously (22). The purity [ $\gamma$-32P]2-azido ATP was determined by TLC analysis.

Photoaffinity labeling was conducted at $4^{\circ} \mathrm{C}$ in $50-\mu 1$ reactions containing $4 \mu \mathrm{g}$ purified recombinant $\mathrm{NBD}, 10 \mathrm{mM}$ tris, $10 \mathrm{mM} \mathrm{MgCl}$, $\mathrm{pH} 7.1$ (without ATP present) and the indicated amount of photoprobe. Reactions were initiated by the addition of photoprobe. After a 30-s incubation, the reactions were photolyzed for $1 \mathrm{~min}$ with a hand-held 254-nm UV lamp $\left(\mathrm{I}=3,100 \mu \mathrm{W} / \mathrm{cm}^{2}\right)$. After photolysis, samples were precipitated with an equal volume of $7 \%$ perchloric acid and resolubilized in a buffer containing 10\% SDS, 3.6 M urea, $162 \mathrm{mM}$ dithiothreitol, $m$-Cresol purple as a tracking dye, and $20 \mathrm{mM}$ Tris- $\mathrm{HCl}, \mathrm{pH}$. 8.0. Samples were then subjected to electrophoresis by an 8-12\% SDSPAGE separating gel with a $4 \%$ stacking gel according to the method of Laemmli (23). Gels were then stained, fixed, and labeling quantified on an AMBIS 4000 Imaging System (Ambis, Inc., San Diego, CA).

\section{Results}

We used prokaryotic over-expression to generate quantities of CFTR NBDs sufficient for binding and structural studies (Fig. 1). Because recombinant proteins synthesized in bacteria (as well as in eukaryotic cells) commonly require refolding protocols to establish binding or other activity, care was taken to 

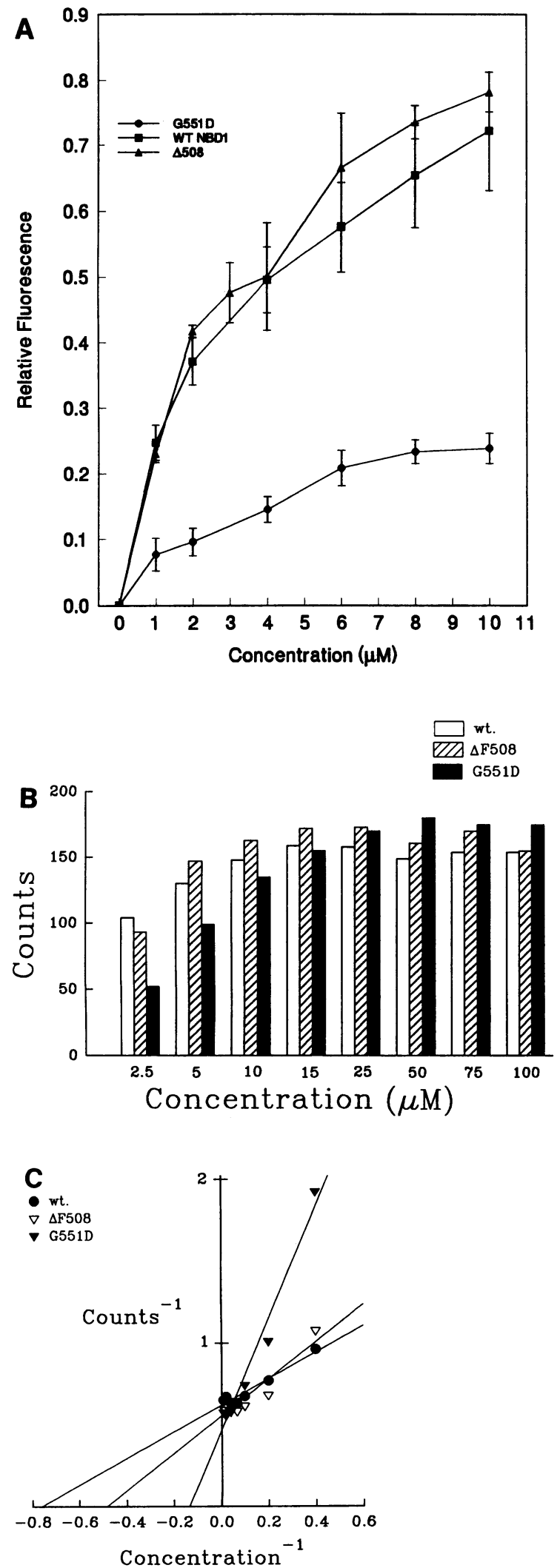

Figure 2. (A) TNP-ATP binding by NBD-1. $250 \mu \mathrm{g} / \mathrm{ml}$ purified NBD-

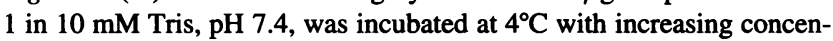
trations of trinitrophenyl ATP. Eadie Hofstee transformation of the above data indicated an apparent half maximal binding at 3.1, 3.5, and $2.9 \mu \mathrm{M}$ for wild type, $\Delta \mathrm{F} 508$, and G551D, respectively; maximal binding values were $0.92,1.05$, and 0.27 relative fluorescence units obtain a refolding sequence relevant to the native CFTR structure and function. First, we have previously shown that recombinant wild-type and $\triangle$ F508 CFTR NBD-1 derived from bacteria bind ATP affinity agarose as well as 32 p-8-azido ATP (9). The data presented below indicates that two additional ATP analogs, trinitrophenyl ATP and 32p-2-azido ATP, also bind the recombinant NBD. All four ligands can be displaced by $\mathrm{Na}_{2}$ ATP with half maximal displacement occurring at $\sim 5 \mathrm{mM}$ ATP. (9, E. J. Sorscher and J. Logan, unpublished observations). Second, the proteins generated for these studies display substantial secondary structural content ( $\sim 70 \% \beta$ sheet, $10 \% \alpha$ helix $)$ when studied by circular dichroism, a result in agreement with a high $\beta$ sheet content reported for a synthetic peptide corresponding to a 67 amino acid segment of the NBD-1 (10). Third, although in vitro studies of recombinant CFTR using cell free membrane patches have demonstrated half maximal ATP stimulation at $\sim 270 \mu \mathrm{M}(4)$, the ATP binding affinity constants which we have observed for NBD-1 are very similar to those reported for CFTR $\mathrm{Cl}^{-}$conductance reported in both native, perfused human sweat ducts and in a non-recombinant human cell line expressing high levels of CFTR (T84) (7, 24; see also 8). Half maximal CFTR activity in both systems was reported at 3-5 mM, a finding which suggested that cellular ATP levels may represent an important regulatory constraint on CFTR anion channel activity. These observations indicate that the nucleotide binding domains used in our studies possess substantial structure after refolding and bind ATP with an affinity very similar to that observed in macroscopic measurements of full length CFTR $\mathrm{Cl}^{-}$conductance.

We used two independent assays of nucleotide binding to test the significance of the $\triangle F 508$ and G551D mutations (Fig. 2). NBD-1 containing the $\Delta$ F508 mutation demonstrated very similar binding to the wild type NBD-1 as measured using either TNP-ATP or 32p-2-azido ATP. This result is consistent with previous studies in our laboratory using other measurements of nucleotide affinity (9), as well as with conclusions reached using fully folded synthetic peptides corresponding to portions of the CFTR NBD-1 with and without F508 (11), and with a computer-based model of the NBD derived from a secondary structural alignment containing the known two- and three-dimensional structure of adenylate kinase (12, see below). In contrast, as shown in Fig. $2 A$, the G551D mutation inhibited

for the three respective proteins. Binding of TNP-ATP by the wild type NBD-1 could be inhibited in the presence of $5 \mathrm{mM} \mathrm{Mg}_{2}$ ATP (50\% inhibition at $5 \mathrm{mM} \mathrm{Mg}_{2}$ ATP). TNP-ATP binding was completely absent in the presence of denaturing concentrations of urea $(4 \mathrm{M})$, indicating the importance of proper folding of the recombinant NBD for nucleotide binding (not shown). $n=7$ for wild type and G551D, $n=3$ for $\triangle$ F508. Error bars $=$ SEM. (B) 32p-2 azido ATP labeling of NBD-1. 32p-2-azido ATP at the concentrations shown was incubated with NBD$1(4 \mu \mathrm{g})$ for $30 \mathrm{~s}$ followed by $1 \mathrm{~min}$ of UV photolysis to covalently label the recombinant proteins within the nucleotide binding site. The gels were stained, fixed, and the labeling quantified on an Ambis 4000 Imaging System (Ambis, Inc.). In $C$, a Lineweaver-Burk plot of the data is shown; half-maximal labeling was obtained at $1.3 \mu \mathrm{M}$ for the wild-type NBD-1, $2.1 \mu \mathrm{M}$ for the $\Delta \mathrm{F} 508 \mathrm{NBD}-1$, and at $7.5 \mu \mathrm{M}$ for NBD-1 containing the G551D mutation. To obtain maximal photolabeling, 32p-2 azido ATP photolysis shown in $B$ and $C$ was performed in the absence of free ATP. 
TNP-ATP fluorescence enhancement by the recombinant domain.

The half maximal TNP-ATP binding values observed for wild type, $\Delta$ F508, and G551D were $3.1,3.5$, and $2.9 \mu \mathrm{M}$, respectively, whereas the maximal binding occurred at 0.92 , 1.05 , and 0.27 relative fluorescent units. We estimated a $5-\mathrm{mM}$ half-maximal ATP binding based on ATP displacement of TNPATP and 32p-2 azido ATP from the CFTR NBD-1. In the NBD, therefore, as in many nucleotide binding proteins, TNP-ATP exhibits several orders of magnitude greater binding affinity than does ATP. This may be due in part to the hydrophobic phenyl group present in TNP-ATP and the hydrophobic nature of many nucleotide binding sites. For example, in the gastric $\mathrm{H}^{+}, \mathrm{K}^{+}$-ATPase, the apparent KD for TNP-ATP binding was $<25 \mathrm{nM}$, whereas the apparent half maximal binding by ATP was $86 \mu \mathrm{M}$ (25). In the $\mathrm{Na} / \mathrm{K}$ ATPase purified from eel electroplax, the KD for TNP-ATP was up to 580 times greater than the KD for ATP, depending on the ionic conditions used (26). Nucleotide binding by a peptide fragment of DNA polymerase I also was substantially tighter for TNP-nucleotides $(\mathrm{kD}=0.5-$ $1.5 \mu \mathrm{M}$ ) than for the corresponding nucleotides without phenyl group attached $(\mathrm{kD}=230-2,100 \mu \mathrm{M})(27)$.

Our estimated binding affinity for ATP agrees closely with our previously reported estimates of ATP affinity for the NBD (9), with macroscipic measurements of the ATP dependence of CFTR $\mathrm{C} 1$ channel activity using two independent systems $(7,24)$ and reasonably well with displacement of TNP-ATP from a maltose binding protein/NBD-1 fusion protein (28), and with displacement of 32p-2 azido ATP from the full length CFTR (29) (half-maximal displacement at $\sim 1 \mathrm{mM}$ ). However, while our results clearly indicate diminished nucleotide binding mediated by the G551D mutation, the micromolar half maximal binding constants using TNP-ATP which we observed are not likely to predict the dependence of CFTR $\mathrm{Cl}^{-}$channel activity on ATP or other nonderivitized nucleotide triphosphates (KD values for ATP dependence of CFTR chloride channel have ranged between $\sim 25 \mu \mathrm{M}$ and $5 \mathrm{mM}$, depending on the particular CFTR $\mathrm{Cl}^{-}$channel preparation used) $(4,5,7$, $24,30)$.

The binding signal obtained with TNP-ATP reflects both the instantaneous number of nucleotide binding sites occupied by the ligand and the local environment within each site (which in turn is reflected in the magnitude of fluorescence enhancement). Because the binding environments within the nucleotide pockets of the wild type, $\Delta$ F508 and G551D NBD-1 polypeptides may not be identical, the quantum of fluorescence enhancement observed for each molecule of TNP-ATP which binds to an NBD molecule cannot be assumed to be the same for the three recombinant NBD-1 proteins. However, even if the fluorescent (quantum) yield for each binding event is different, if the same number of binding sites exist in solution, the ligand (TNP-ATP) concentration observed at saturation should not vary between the three NBD-1 polypeptides. This is not the case in our experiments (Fig. $2 \mathrm{~A}$ ), where G551D saturates at 8-10 $\mu \mathrm{m}$ TNP-ATP, and saturation for the wild-type and the $\triangle F 508$ NBD occurred at significantly higher concentrations. This results suggests that in addition to physically disrupting the nucleotide binding pocket within the nucleotide binding site (see Discussion), the $\mathrm{G} \rightarrow \mathrm{D}$ replacement at position $551 \mathrm{might}$ also destabilize overall protein folding in such a way as to create an equilibrium between NBD folded and unfolded forms. The result would be an apparent decrease in measurable binding sites within the NBD preparation and would explain the lower ligand concentration at saturation for G551D described in Fig. 2 A. Ultraviolet spectral analysis of the structures of wild-type and mutant NBDs in the presence and absence of nucleotides, and in the presence of denaturants such as urea $(9,11)$ are in progress to evaluate effects on protein folding stability mediated by G551D.

To confirm that less nucleotide was actually bound to CFTR polypeptides containing the G551D mutation, we performed an additional series of experiments using an independent nucleotide binding assay. As shown in Fig. 2, $B$ and $C$, at concentrations below saturation, 32p-2-azido ATP labeling is diminished in the G551D mutant as compared with wild type (half-maximal photoinsertion was [in $\mu \mathrm{M}] 1.3$ [wt]; 2.1 [ $\Delta \mathrm{F} 508$ ]; and 7.5 [G551D]. Because the two assays used in this study measure complementary features of nucleotide binding environment and ligand occupancy, taken together these results indicate defective nucleotide binding mediated by the G551D mutation. While the fluorescence enhancement observed for TNP-ATP binding depends primarily upon the integrity and local environment of a nucleotide binding pocket, $32 \mathrm{p}-2$ azido ATP labeling not only requires an adequate binding site, but also requires properly oriented residues within the binding site which are accessible for covalent photolabeling. In our experiments, the functional defect caused by G551D led to an apparent decreased maximal binding by TNP-ATP (Fig. $2 A$ ), and a diminished half maximal binding in experiments using the $32 \mathrm{p}-2$ azido ATP (Fig. $2 B$ ). Such an observation could be explained by a change in nucleotide binding pocket hydrophobicity (causing a decreased fluorescence signal from TNP-ATP even at maximal occupancy) together with loss of one of a small number of sites normally available for covalent photolabeling by 32p-2-azido ATP (an abnormality with might be overcome at increasing concentrations of photoaffinity ligand).

Although the glycine corresponding to G551 is invariant among more than $30 \mathrm{ABC}$ proteins (and an even larger numbers of NBDs, since each ABC protein possesses 2 NBDs), the functional significance of this glycine residue and the immediately adjacent, highly conserved residues found within all members of the gene family is not known (Other members of the gene family, for example, include the human multidrug resistance gene (MDR), the chloroquine resistance gene product of $P$. malaria, and the recently described human adrenoleukodystrophy gene $[1,12,13,31-34])$. An LSGGQXQR motif is highly conserved across the ABC gene family (glycine corresponding to G551 underlined) $(1,12,13)$, but is not present in other nucleotide binding proteins such as adenylate kinase, for example, or included within the so-called Walker A or Walker B sequences characterizing a broad range of non-ABC family, ATP-binding proteins $(35,36)$. It has been proposed that in the histidine permease of $S$. typhimurium (an ABC protein), the residues near the LSGGQXQR motif could serve as a "linker peptide"' which conveys conformational changes between segments of the domain (37).

To further test our hypothesis that this conserved region functions in nucleotide binding, we generated additional recombinant proteins corresponding to the wild-type CFTR NBD-2 and to NBD-2 containing a G1349D mutation (i.e., a mutation in NBD-2 which causes clinical CF and occurs at the glycine residue in NBD-2 which aligns with G551 in NBD-1). As shown in Fig. 3, G1349D disrupts binding both of TNP-ATP and 32p-2-azido ATP. Maximal binding values observed with 

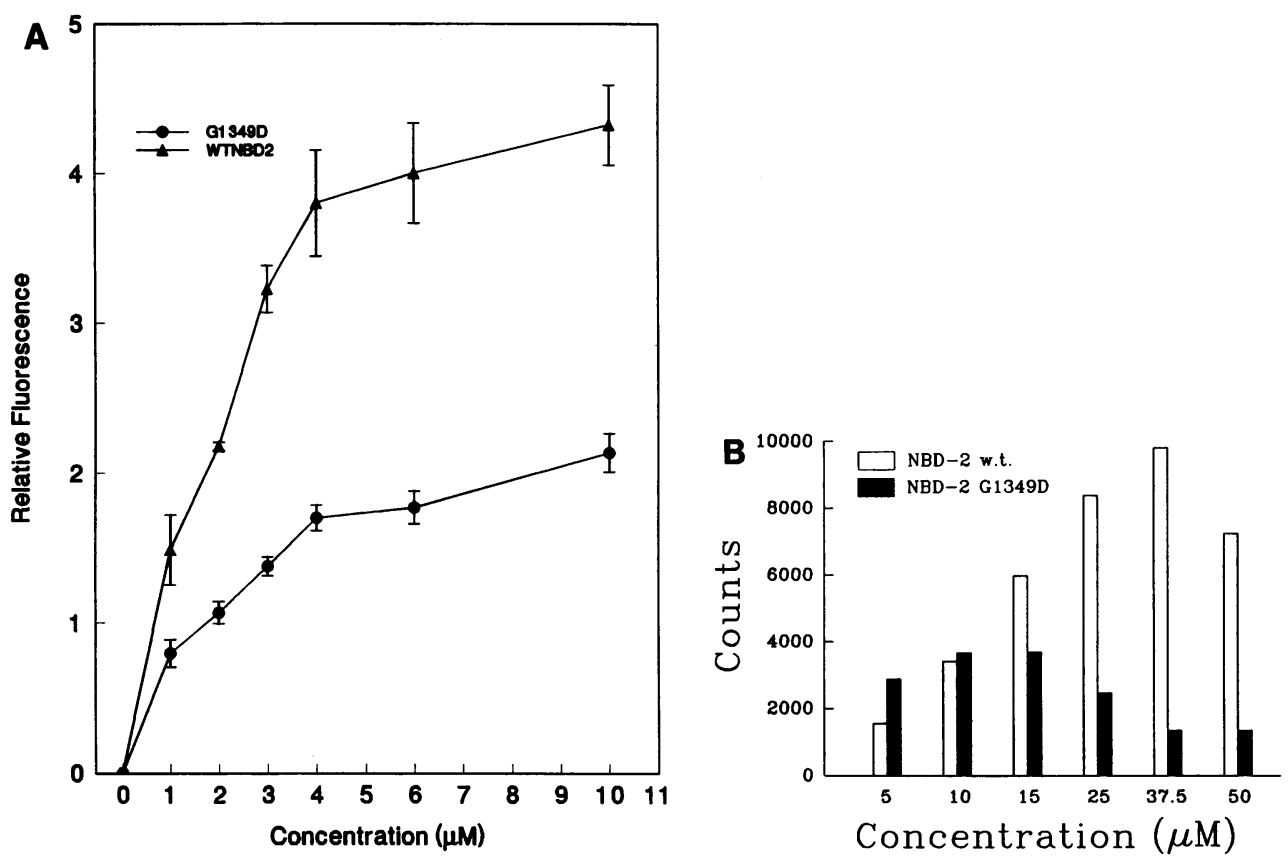

Figure 3. Binding of nucleotide by CFTR NBD-2. $A$ and $B$ show TNP-ATP binding and 32p-2 azido ATP labeling of the wild-type and G1349D NBD-2, respectively. Experiments were performed as in Fig. 2. Maximal binding of TNP-ATP to wild-type NBD-2 was $\sim 2.5 \times$ greater than that observed for the G1349D mutant. (Using algebraic transformation as described in the previous figure, for wt NBD-2, maximal binding $=8.9$ fluorescence units; half maximal binding $=3.8 \mu \mathrm{M}$ TNP-ATP; for G1349D, maximal binding $=3.5$ fluorescence units, half maximal at $5.3 \mu \mathrm{M}$ TNP-ATP). As shown in $B$, inclusion of G1349D within NBD-2 blocked azido nucleotide labeling of the protein. The magnitude of 32p-2 azido ATP labeling is highly dependent upon the specific activity of the photoaffinity ligand used. The specific activity of 32p-2 azido ATP used in Fig. $3 B$ was $\sim 10$-fold higher than that used in Fig. $2 B$. When wild-type NBD-1 and NBD-2 were directly compared using photolabeling under identical conditions (and using the same lot of 32p-2 azido ATP), a 10-fold enhancement of maximal photolabeling was observed in NBD-2 compared with NBD-1 (data not shown).

TNP-ATP were 8.9 fluorescence units (wild-type NBD-2) and 3.5 fluorescence units (G1349D NBD-2); half-maximal binding was observed at 3.8 and $5.3 \mu \mathrm{m}$ TNP-ATP for the wt NBD-2 and G1349D mutants, respectively. Half-maximal photoinsertion of the wild type NBD-2 with 32p-2 azido ATP was observed at a ligand concentration of $\sim 13 \mu \mathrm{m}$, whereas photolabeling was substantially less for the G1349D compared with the wild-type NBD-2 at every ligand concentration studied. These findings have significance, since they suggest a general function of the conserved glycine and surrounding residues may be in nucleotide binding. The results are consistent both with previous observations indicating an abnormal ATP dependence in CFTR containing mutations in this glycine residue (5) and with the observed loss of function of other $\mathrm{ABC}$ proteins containing comparable mutations (38). However, experiments measuring CFTR or other $A B C$ protein function could not distinguish between disrupted ATP binding, hydrolysis, or utilization of nucleotide as the mechanism of observed abnormalities in function. Our data support the notion that in addition to abnormal biosynthetic processing, cystic fibrosis may result from defects in nucleotide binding by the CFTR. It is likely that an additional mechanism (i.e., absent CFTR polypeptide due to the presence of premature stop codons within certain mutant CFTR alleles) may also account for the defect in some cases $(18,39)$.

\section{Discussion}

To test the influence of CF mutations on nucleotide binding, we constructed and purified the CFTR domains which have been implicated as both necessary and sufficient for nucleotide interactions with CFTR (1-5, 9-13). The highly conserved, functionally independent and even interchangeable nature of CFTR and other nucleotide binding domains (which span $\sim 200$ amino acids and share $30 \%$ amino acid identity throughout the $\mathrm{ABC}$ gene family) suggests that one approach to understanding the molecular pathogenesis of CFTR mutations might focus on the domains themselves, as opposed to any particular ATP binding cassette protein context. For example, the NBDs in the LIVI neutral branched chain amino acid transport system of $E$. coli and the histidine permease of $S$. typhimurium are found on genes separate from the remainder of the transport complex (and are therefore synthesized separately), but assemble with the remainder of the $\mathrm{ABC}$ complex at the cell membrane. When CFTR NBD mutations were placed at corresponding residues within these bacterial NBDs, (or within the NBDs of the yeast mating factor transporter, STE 6), certain of these mutations had functional effects consistent with those observed in CFTR (although others did not. See refs. 37, 38, 40). Furthermore, in $\mathrm{ABC}$ proteins containing both NBDs within a single polypeptide chain, the two NBDs can be separated and still maintain function. For example, when the STE 6 cDNA, which codes for both NBDs of the protein, was severed into two halves, (each of which contained one of the NBDs ), coexpression of the two STE 6 truncations led to functional reconstitution of STE 6 mediated a-factor transport (40).

NBDs are also interchangeable among members of the ABC protein family. The NBD-1 of CFTR, for example, can be grafted into STE 6 in place of the STE 6 NBD-1 to allow 
functional transport of mating factor. Importantly, NBD-1 mutations known to disrupt CFTR function also led to disrupted mating factor transport by the chimeric STE 6/CFTR protein (41). Finally, although data concerning a requirement for ATP hydrolysis in CFTR activity is not yet conclusive, in some members of the ATP binding cassette gene family known to hydrolyze ATP, isolated NBDs are sufficient to mediate this hydrolysis $(12,42,43)$. Taken together, these findings demonstrate that the NBDs within the ATP binding cassette family are functionally independent, self-contained "cassettes" and emphasize $(a)$ that characterization of the nucleotide binding domains, themselves, (independent of the transmembrane, regulatory and other regions within ATP binding cassette proteins) can offer substantial insight into the mechanism of action within this family of gene products, and $(b)$ the relevance of normal and abnormal function of the domains to the activity of the full length $A B C$ proteins. Indeed, in a similar approach to that applied in the current study, binding and structural studies of synthetic peptides corresponding to portions of the CFTR NBD$1(10,11)$, as well as computer-assisted models of isolated nucleotide binding domains based on secondary structural alignments with the known crystal structures of adenylate kinase, p21 ras, and the elongation factor Tu, have previously been used to predict the significance of clinically important mutations on CFTR nucleotide binding and function (see below, 12, 13). NBDs within the ABC family very likely subserve similar functions, so that conclusions concerning the CFTR NBD-1 could be applicable to CFTR NBD-2 and to NBDs of other members of the gene family as well.

The maximal binding of TNP-ATP by wild type and $\triangle F 508$ NBD-1 was 3-4-fold greater than that observed for the G551D mutant. Defective maximal binding capacity of a similar magnitude has been proposed to account for some forms of familial hypercholesterolemia (in which ligand binding by mutant LDL receptor is decreased by fourfold) (44), for oxygen affinity mutants in clinical hemoglobinopathies (45), as well as in the twofold difference in binding or enzymatic function which accounts for enzymatic defects of many congenital diseases (e.g., those inherited in an autosomal dominant fashion) (46). In addition, since in most cases the $G \rightarrow D$ mutant allele causes disease in conjunction with a $\Delta \mathrm{F} 508$ allele, nucleotide binding in a compound heterozygote (which may already be $50 \%$ decreased due to aberrant processing of the $\Delta \mathrm{F} 508$ gene product) might represent only a residual of $15-20 \%$ of that seen in a wild-type cell.

Although the anion channel activity of wild-type and mutant CFTR polypeptides has become the most useful endpoint defining normal CFTR function, mutations which cause clinical CF in many cases cause only small alterations in channel activity in vitro. For example, in a series of four NBD-1 and NBD-2 mutations which cause disease (including G1349D and G551S), although 4-8-fold differences in open probability were observed in vitro when compared with wild type, no abnormalities in maximal single channel currents could be documented (although small differences in the dependence of current on ATP were observed for the mutants) (5). In vivo, potential difference measurements in respiratory epithelia of CF patients differ by less than threefold compared with normal controls $(47,48)$. This data further supports the notion that even subtle changes in normal CFTR function may have important physiologic consequences. Finally, measurements of CFTR activity in intact human tissues and cell monolayers have indicated that half maximal CFTR activation requires maximal cellular ATP levels $(\sim 5 \mathrm{mM})$, and that CFTR activity may be coupled to physiologic nucleotide levels within the cell $(7,24)$. The decrease in binding of ATP analogs demonstrated here has significance, therefore, since a similar defect in nucleotide binding in vivo could result in mutant CFTR which would be activated only at nucleotide levels which are not present within the cell.

Our studies using recombinant CFTR NBDs provide a test of recent predictions concerning the function and structure of NBDs within the $A B C$ gene family. For example, based upon Michaels-Menten analyses of the ATP dependence of CFTR anion channel open probability, it has been proposed both that the full length CFTR contains only one functional ATP binding site (30), and that two active nucleotide binding sites are present within the full length molecule (5). Our results are the first to directly demonstrate that both CFTR NBD-1 and NBD-2 are capable of binding nucleotide, and therefore provide evidence for the presence of more than one functional CFTR nucleotide binding site. In addition, binding affinity appears to be different between the two domains. NBD-2 exhibits a modest (ninefold) increase in maximal TNP-ATP fluorescence when compared with NBD-1 (compare Figs. $2 A$ and $3 A$ ), and an approximate 10 -fold increase in photolabeling with $32 \mathrm{p}-2$ azido ATP when NBD-2 and NBD-1 are compared in the same experiment (data not shown). Increased affinity of NBD-2 for a nucleotide analog could support the notion that two NBDs of CFTR may not have identical roles in the maintenance of CFTR $\mathrm{Cl}^{-}$channel activity. The possibility of different functions of the two CFTR NBDs has been suggested previously based upon studies of the influence of NBD-1 and NBD-2 mutations on the CFTR $\mathrm{Cl}^{-}$channel in isolated membrane patches, where functional differences in the domains led to speculation that ATP hydrolysis by CFTR was dependent primarily on NBD-2 rather than NBD-1 (5).

Although a high resolution crystal structure is not yet available for any of the ABC protein NBDs, Chou-Fasman based predicted secondary structure of the CFTR and other NBDs corresponds very closely to that of non-ABC, known nucleotide binding proteins such as adenylate kinase, p21 ras, and the elongation factor, Tu. The striking alignment of the predicted $2^{\circ}$ structure of the CFTR NBD and the known structure of these other proteins has been used to predict the functional consequences of disease-associated CFTR mutations. For example, a model of the CFTR NBD based on $2^{\circ}$ structural homology with adenylate kinase (12) predicted that the $\Delta F 508$ mutation would be spatially so distant from the nucleotide binding pocket of CFTR NBD-1 that it would not disrupt ATP binding. Our results are consistent with this prediction. No specific prediction was made concerning the $\mathrm{G} \rightarrow \mathrm{D}$ mutations at CFTR positions 551 and 1349. In an attempt to relate NBD tertiary structural models to our findings concerning nucleotide binding, assignment of the corresponding locations of these residues within the known adenylate kinase tertiary structure was performed.

As shown in Fig. 4, assignment of the positions of G551 and G1349 is straightforward based on previously determined alignments (12). These residues would be predicted to fall immediately after the third $\beta$ strand in both the adenylate kinase and the predicted CFTR NBD structure. In addition, in the original CFTR NBD model, the ATP binding pocket was assigned to a large cleft corresponding to the AMP binding site in adenylate kinase. When this binding site is appropriately adjusted based on the most recent crystallographic and NMR data available for the location of the AMP binding site in ade- 

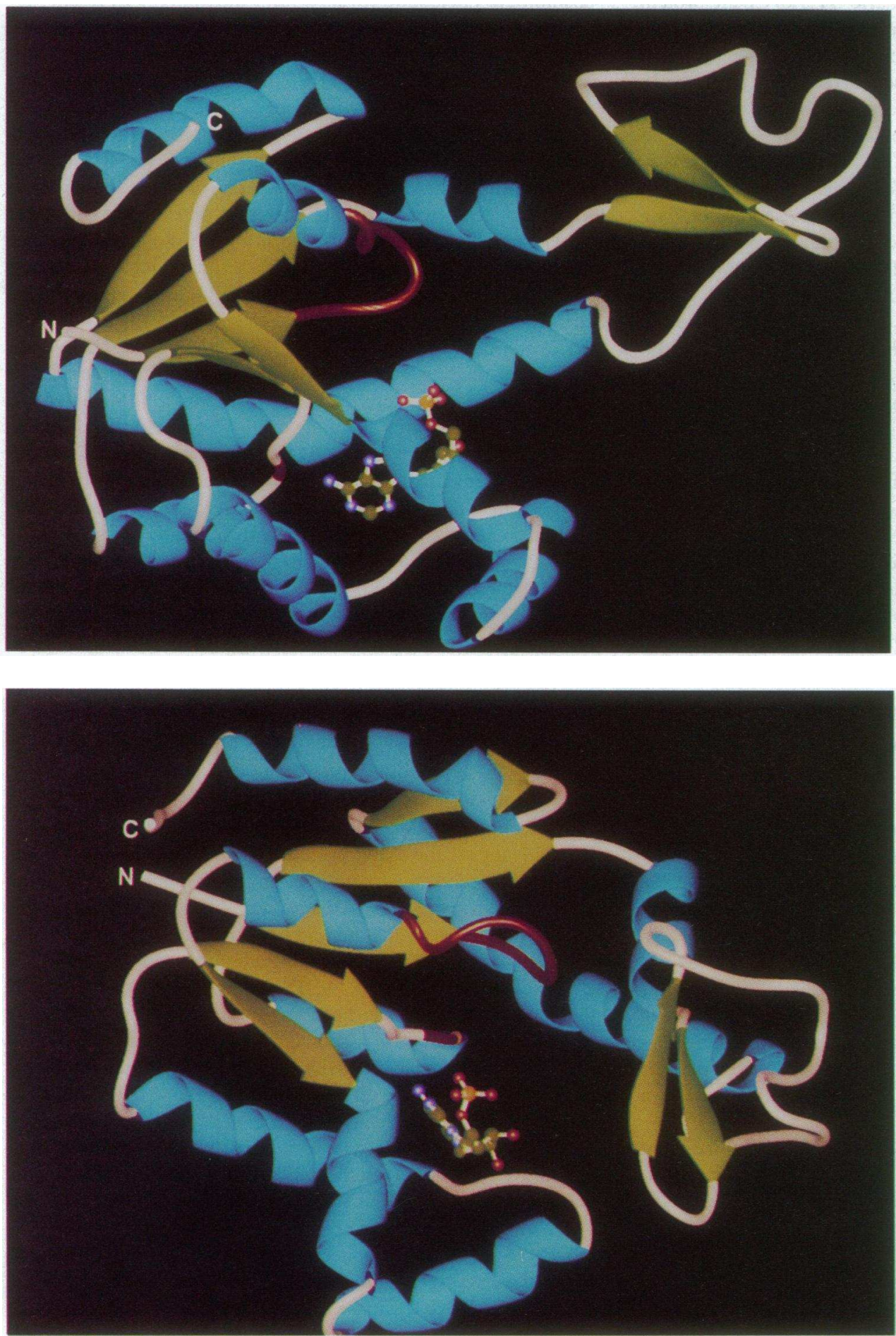

Figure 4. Positions of residues G551 and G1349 in a tertiary structural model of the NBD based on adenylate kinase. The similarities between predicted secondary structure of $\mathrm{ABC}$ protein NBDs and the known structure of adenylate kinase, together with the structural similarities of nucleotide binding sites within diverse, unrelated proteins outside of the $\mathrm{ABC}$ family (e.g., between adenylate kinase, the elongation factor $\mathrm{Tu}$ and $\mathrm{p} 21$ ras), suggest that the tertiary structure of nucleotide binding sites in the NBDs of CFTR and other ABC family members may closely resemble those of adenylate kinase $(12,13$, 35 ). In an attempt to relate previous tertiary structural models of CFTR to our findings of defective nucleotide binding by the G551D and G1349D NBDs, we predicted the location of these residues based on previous alignments of CFTR NBDs with adenylate kinase. As shown in the Figure, G551 and G1349 occur in a loop immediately following the third $\beta$ strand of both the adenylate kinase and the CFTR NBD models (49, 50 ; the prediction also conforms to the CFTR NBD model of ref. 12). The Walker A sequence is part of a connecting loop between the first $\beta$ strand and an $\alpha$ helix. Both the Walker A motif and the residues at G551 or G1349 are predicted to fall within a large cleft suitable for nucleotide binding; this cleft is known both by NMR and $x$-ray crystallography to bind AMP within the adenylate kinase molecule. The two views above are $\sim 90^{\circ}$ rotated from each other; the $\beta$ strands are green, $\alpha$ helices are blue, and the loops are white. The Walker A site and the location of G551 or G1349 are shown in red. A ball and stick model of AMP is shown in a nucleotide binding site. The atoms are colored according to the following code: $\mathrm{N}$, blue, $\mathrm{O}$, red, $\mathrm{C}$, green, $\mathrm{P}$, yellow. The figure was prepared using the program Ribbon $2.0(51)$. nylate kinase $(49,50)$, as shown in Fig. 4, G551 and G1349 are on the wall of a cleft suitable for ATP binding within the NBD. Another wall of the cleft is formed by the so-called ploop or Walker A motif (GXXXXGKT/S) which is $(a)$ present in nearly all ATP binding proteins, $(b)$ known to contain residues which actually coordinate with phosphate groups of ATP ( such as the p-loop lysine within p21 ras), and $(c)$ highly likely to be involved in ATP binding by the CFTR and other NBDs, since the conservation of this motif is invariant through the ABC gene family $(1,12,35,36)$. Recent data from our laboratories has also shown that a peptide containing residues 1335 to 1351 is covalently labeled in the intact NBD-2 by $32 \mathrm{p}-2$ azido ATP, suggesting the proximity of this region to the ATP binding site within the NBD. Thus our findings of diminished 
nucleotide binding mediated by G551D and G1349D, as well as normal binding by $\Delta F 508$, provide experimental evidence supporting predictions of the structure of the ATP binding site within the CFTR NBD. The results further support the notion that the LSGGQXQR motif found within all $A B C$ proteins is involved in normal nucleotide binding, and in fact may form a critical portion of an ATP binding cleft. An essential role in nucleotide binding could explain the high level of conservation of this region within the $\mathrm{ABC}$ gene family, and would offer a molecular mechanism by which G551D and G1349D contribute to clinical CF.

\section{Acknowledgments}

The authors wish to thank Dr. Richard Gregory and Dr. Alan Smith for providing the G551D and G1349D mutant CFTR cDNAs used in this study. We also wish to thank Dr. Sadis Matalon for helping to establish the TNP-ATP protocol, and Dr. M.-D. Tsai for useful discussions concerning the adenylate kinase structural model. We are grateful to Dr. Tipo Liu and Edward Walthall for assistance with the Figures, and to Dr. Shenyi Peng and Yuee Wang for technical assistance. We thank Jennifer Smith and Bonnie Parrott for excellent secretarial assistance.

This work was supported by National Institutes of Health grant P01 DK38518 and by the Cystic Fibrosis Foundation. Eric J. Sorscher is a Lucille P. Markey Scholar in the Biomedical Sciences.

\section{References}

1. Riordan, J. R., J. M. Rommens, B.-S. Kerem, N. Alon, R. Rozmahel, Z. Grzelczak, J. Zielenski, S. Lok, N. Plavsic, J.-L. Chou, M. L. Drumm, M. C. Iannuzzi, F. S. Collins, and L.-C. Tsui. 1989. Identification of the cystic fibrosis gene: Cloning and characterization of complementary DNA. Science (Wash. DC). 245:1066-1073.

2. Tsui, L. -C. 1991. Probing the basic defect in cystic fibrosis. Current Opinion in Genetics and Development. 1:4-10.

3. Collins, F. S. 1992. Cystic fibrosis: Molecular biology and therapeutic implications. Science (Wash. DC). 256:774-779.

4. Anderson, M. P., H. A. Berger, D. P. Rich, R. J. Gregory, A. E. Smith, and M. J. Welsh. 1991. Nucleotide triphosphates are required to open the CFTR chloride channel. Cell. 67:775-784.

5. Anderson, M. P., and M. J. Welsh. 1992. Regulation by ATP and ADP of CFTR chloride channels that contain mutant nucleotide-binding domains. Science (Wash. DC). 257:1701-1704.

6. Bear, C. E., C. Li, and N. Kartner, R. J. Bridges, T. J. Jensen, M. Ramjeesingh, and J. R. Riordan. 1992. Purification and functional reconstitution of the cystic fibrosis transmembrane conductance regulator (CFTR). Cell. 68:809-818.

7. Quinton, P. M., and M. M. Reddy. 1992. Control of CFTR chloride conductance by ATP levels through non-hydrolytic binding. Nature (Lond.). 360:79-81.

8. Wine, J. J., and S. C. Silverstein. 1992. ATP and chloride conductance. Nature (Lond.). 360:18.

9. Hartman, J., Z. Huang, T. A. Rado, S. Peng, T. Jilling, D. D. Muccio, and E. J. Sorscher. 1992. Recombinant synthesis, purification, and nucleotide binding characteristics of the first nucleotide binding domain of the cystic fibrosis gene product. J. Biol. Chem. 267:6455-6458.

10. Thomas, P. J., P. Shenbagamurthi, X. Ysern, and P. L. Pedersen. 1991. Cystic fibrosis transmembrane conductance regulator: Nucleotide binding to a synthetic peptide. Science (Wash. DC). 251:555-557.

11. Thomas, P. J., J. S. Shenbagamurthi, J. M. Hullihen, and P. L. Pederson 1992. Effects of the most common cystic fibrosis-causing mutation on the secondary structure and stability of a synthetic peptide. J. Biol. Chem. 267:5727-5730.

12. Hyde, S., P. Emsley, M. J. Hartshorn, M. M. Mimmack, U. Gileadi, S. R. Pearce, M. P. Gallagher, D. R. Gill, R. E. Hubbard, and C. F. Higgins. 1990 Structural model of ATP-binding proteins associated with cystic fibrosis, multidrug resistance and bacterial transport. Nature (Lond.). 346:362-365.

13. Mimura, C. S., S. R. Holbrook, and G. F. Ames. 1991. Structural model of the nucleotide-binding conserved component of periplasmic permeases. Proc. Natl. Acad. Sci. USA. 88:84-88.

14. Cheng, S. H., R. J. Gregory, J. Marshall, S. Paul, D. W. Souza, G. A. White, C. R. O'Riordan, and A. E. Smith. 1990. Defective intracellular transport and processing of CFTR is the molecular basis of most cystic fibrosis. Cell. 63:827-834.

15. Gregory, R. J., D. P. Rich, S. H. Cheng, D. W. Souza, S. Paul, P. Manavalan, M. P. Anderson, M. J. Welsh, and A. Smith. 1991. Maturation and function of cystic fibrosis transmembrane conductance regulator variants bearing mutations in putative nucleotide-binding domains 1 and 2. Mol. Cell. Biol. 11:3886-3893.

16. Denning, G. M., M. P. Anderson, J. F. Amara, J. Marshall, A. E. Smith, and M. W. Welsh. 1992. Processing of mutant cystic fibrosis transmembrane conductance regulator is temperature-sensitive. Nature (Lond.). 358:761-710.

17. Kartner, N., O. Augustinas, T. J. Jensen, A. L. Naismith, and J. R. Riordan. 1992. Mislocalization of $\triangle F 508$ CFTR in cystic fibrosis sweat gland. Nature Genetics. 1:321-327.

18. Tsui, L-C. 1992. The spectrum of cystic fibrosis mutations. Trends in Genet. 8:392-398.

19. Richards, B., J. Skoletsky, A. P. Shuber, R. Balfour, R. C. Stern, H. L. Dorkin, R. B. Parad, D. Witt, and K. W. Klinger. 1993. Multiplex PCR amplification from the CFTR gene using DNA prepared from buccal brushes/swabs. Hum. Mol. Gen. 2:159-163.

20. Kim, H., and B. Haley. 1990. Synthesis and Properties of 2-Azido-NAD ${ }^{+}$ J. Biol. Chem. 265 P. 3636-3641.

21. Salvucci, M. E., A. J. Chavan, and B. E. Haley. 1992. Identification of peptides from the adenine binding domains of ATP and AMP in adenylate Kinase: isolation of photoaffinity-labeled peptides by metal chelate chromatography. Biochemistry. 31:4479-4487.

22. Potter, R. L., and B. E. Haley. 1983. Photoaffinity Labeling of Nucleotide Binding Sites with 8-azidopurine Analogs. Methods Enzymol. 91:613-663.

23. Laemmli, U. K. 1970. Cleavage of structural proteins during the assembly of the head of Bacteriophage 4. Nature (Lond.). 227:680-685.

24. Bell, C. L., and P. M. Quinton. 1993. Regulation of CFTR Cl conductance in secretion by cellular energy levels. Am. J. Physiol. 264:C925-C931.

25. Faller, L. D. 1989. Competitive binding of ATP and the fluorescent substrate analogue $2^{\prime}, 3^{\prime}-0$-(2.4.6-Trinitropenylcyclohexadienylidine) adenosine $5^{\prime}$ Triphosphate to the gastric $\mathrm{H}^{+}, \mathrm{K}^{+}$-ATPase: evidence for two classes of nucleotide sites. Biochemistry. 28:6771-6778.

26. Moczydlowski, E. G., and P. A. G. Fortes. 1981. Characterization of $2^{\prime}, 3^{\prime}$ 0 -(2,4,6-trinitrocyclohexadienylidine) adenosine $5^{\prime}$-triphosphate as a fluorescent probe of the ATP site of sodium and potassium transport adenosine triphosphatase. J. Biol. Chem. 256:2346-2356.

27. Mullen, G. P., P. Shenbagamurthi, and A. S. Mildvan. 1989. Substrate and DNA binding to a 50-residue peptide fragment of DNA polymerase I. $J$. Biol. Chem. 264:19637-19647.

28. Ko, Y. H., P. J. Thomas, M. R. Delannoy, and P. L. Pedersen. 1993. The cystic fibrosis conductance regulator. Overexpression, purification, and characterization of wild type and $\Delta$ F508 mutant forms of the first nucleotide binding fold in fusion with the maltose-binding protein. J. Biol. Chem. 15:24330-24338.

29. Travis, S. M., M. R. Carson, D. R. Ries, and M. J. Welsh. 1993. Interaction of nucleotides with membrane-associated cystic fibrosis transmembrane conductance regulator. J. Biol. Chem. 268, 21:15336-15339.

30. Schultz, B. D., C. J. Venglaric, R. J. Bridges, and R. A. Frizzell. 1993. Regulation of CFTR $\mathrm{Cl}^{-}$channels by adenine nucleotide phosphates. FASEB (Fed. Am. Soc. Exp. Biol.) J. 7:2464 (Abstr.).

31. Ambudkar, S. V., I. H. Lelong, J. Zhang, C. O. Cardarelli, M. M. Gottesman, and I. Pastan. 1992. Partial purification and reconstitution of the human multidrug-resistance pump: Characterization of the drug-stimulatable ATP hydrolysis. Proc. Natl. Acad. Sci. USA. 89:8472-8476.

32. Gill, D. R., S. C. Hyde, C. F. Higgins, M. A. Valverde, G. M. Mintenig, and F. V. Sepulveda. 1992. Separation of drug transport and chloride channel functions of the human multidrug resistance P-glycoprotein. Cell. 71:23-32.

33. Sarkadi, B., E. M. Price, R. C. Boucher, U. A. Germann, and G. A. Scarborough. 1992. Expression of the human multidrug resistance cDNA in insect cells generates a high activity drug-stimulated membrane ATPase. J. Biol. Chem. 267:4854-4858.

34. Mosser, J. A-M. Douar, C-O. Sarde, P. Kioschis, R. Feil, H. Moser, A-M. Poustka, J-L. Mandel, and P. Aubourg. 1993. Putative x-linked adrenoleukodystrophy gene shares unexpected homology with $\mathrm{ABC}$ transporters. Nature (Lond.). 361:726-730.

35. Saraste, M., P. R. Sibbald, and A. Wittinghofer. 1990. The P-loop-a common motif in ATP- and GTP-binding proteins. TIBS (Trends Biochem. Sci.). 15:430-434.

36. Walker, J. E., M. Saraste, M. J. Runswick, and N. J. Gar. 1982. Distantly related sequences in the $\alpha$ - and $\beta$-subunits of ATP synthase, myosin, kinases and other ATP-requiring enzymes and a common nucleotide binding fold. $E M B O$ (Eur. Mol. Biol. Organ.) J. 8:945-951.

37. Shyamala, V., V. Baichwal, E. Beall, and G. Ames. 1991. Structurefunction analysis of the histidine permease and comparison with cystic fibrosis mutations. J. Biol. Chem. 266:18714-18719.

38. Gibson, A. L., L. M. Wagner, F. S. Collins, and D. L. Oxender. 1991. A bacterial system for investigating transport effects of cystic fibrosis-associated mutations. Science (Wash. DC). 254:109-111.

39. Cutting, G. R., L. M. Kasch, B. J. Rosenstein, L.-C. Tsui, H. H. Kazazian, Jr., and S. E. Antonarakis. 1990. Two patients with cystic fibrosis, nonsense mutations in each cystic fibrosis gene, and mild pulmonary disease. N. Engl. J. Med. 323:1685-1689.

40. Berkower, C. and S. Michaelis. 1991. Mutational analysis of the yeast a- 
factor transporter STE6, a member of the ATP binding cassette (ABC) superfamily. EMBO (Eur. Mol. Biol. Organ.) J. 10:3777-3785.

41. Teem, J. L., H. A. Berger, L. S. Ostedgaard, D. P. Rich, L.-C. Tsui, and M. J. Welsh. 1993. Identification of revertants for the cystic fibrosis $\Delta F 508$ mutation using STE6-DFTR chimeras in yeast. Cell. 73:335-346.

42. Bishop, L., R. Agbayani, Jr., S. V. Ambudkar, P. C. Maloney, and G. F.-L. Ames. 1989. Reconstitution of a bacterial periplasmic permease in proteoliposomes and demonstration of ATP hydrolysis concomitant with transport. Proc. Natl. Acad. Sci. USA. 86:6953-6957.

43. Mimack, M. L., M. P. Gallagher, S. R. Pearce, S. C. Hyde, I. R. Booth, and C. F. Higgins. 1989. Energy coupling to periplasmic binding protein-dependent transport systems: Stoichiometry of ATP hydrolysis during transport in vivo. Proc. Natl. Acad. Sci. USA. 86:8257-8261.

44. Lehrman, M. A., J. L. Goldstein, D. W. Russell, and M. S. Brown. 1987. Duplication of seven exons in LDL receptor gene caused by Alu-Alu recombination in a subject with familial hypercholesterolemia. Cell. 48:827835.

45. Bunn, H. F., and B. G. Forget. 1986. Hemoglobinopathy due to abnormal oxygen binding. In Hemoglobin: Molecular, Genetic and Clinical Aspects, W. B. Saunders, Inc. editors. Philadelphia, PA. 595-622.

46. McKusick, V. 1989. Genetic map of the human genome autosomes, and, $\mathrm{x}, \mathrm{y}$, and mitochondrial chromosomes. In The Metabolic Basis of Inherited Diseases, 6th ed. C. R. Scriver, A. L. Beaudet, W. S. Sly, and D. Valle, editors. New York. 73-163.

47. Knowles, M., J. Gatzy, and R. Boucher. 1981. Increased bioelectric potential difference across respiratory epithelia in cystic fibrosis. N. Engl. J. Med. 305:1489-1495.

48. Knowles, M. R., L. L. Clarke, and R. Boucher. 1991. Activation by extracellular nucleotides of chloride secretion in the airway epithelia of patients with cystic fibrosis. N. Engl. J. Med. 325:533-538.

49. Diederichs, K., and G. E. Schulz. 1990. Three-dimensional structure of the complex between the mitochondrial matrix adenylate kinase and its substrate AMP. Biochemistry. 29:8138-8144.

50. Tsai, M.-D., and H. Yan. 1991. Mechanism of adenylate kinase: Sitedirected mutagenesis versus X-ray and NMR. Perspect. Biochem. 30:6806-6818.

51. Carson, M. 1987. Ribbon models of macromolecules. J. Mol. Graphics. 5:103-106. 